\title{
Refreshing Labioplasty Techniques for Plastic Surgeons
}

\author{
Bassem Nathan • Lawrence Mascarenhas
}

Received: 24 November 2012/ Accepted: 31 January 2013/Published online: 16 February 2013

(C) Springer Science+Business Media New York and International Society of Aesthetic Plastic Surgery 2013

Level of Evidence $V$ This journal requires that authors assign a level of evidence to each article. For a full description of these Evidence-Based Medicine ratings, please refer to the Table of Contents or the online Instructions to Authors www.springer.com/00266.

We comment on the review of labioplasty by Drs. Triana and Robeldo [1] The authors studied 74 patients and reviewed them during an average follow-up period of 6 months according to a prescribed follow-up protocol of seven postoperative visits.

Although we have not been informed how many of their patients returned for follow-up, their experience seems to be at odds with ours because less than $5 \%$ of our labioplasty patients keep their second postoperative follow-up appointment. We wonder whether the authors have an explanation for why satisfied patients would return seven times for a follow-up evaluation and whether the authors enjoy a special cultural advantage.

Also, the authors state that their patients ranged in age from 23 to 47 years and that two of their photographed patients were ages 52 and 58 years, respectively. We assume this was an error. Finally we commend the authors for their accumulated experience and their excellent photographic follow-up evaluation.

\section{Reference}

1. Triana L, Robeldo AM (2012) Refreshing labioplasty techniques for plastic surgeons. Aesthetic Plast Surg 36:1078-1086

B. Nathan $(\bowtie)$

Riverside Hospital, Brentside Executive Park,

London TW8 9DR, UK

e-mail: bassem@doctors.net.uk

L. Mascarenhas

Guy's and St. Thomas's Hospitals, London, UK 